\title{
Pushing the limits of NuSTAR detectors
}

Brian W. Grefenstette, W. Rick Cook, Fiona A. Harrison, Takao Kitaguchi, Kristin K. Madsen, et al.

Brian W. Grefenstette, W. Rick Cook, Fiona A. Harrison, Takao Kitaguchi, Kristin K. Madsen, Hiromasa Miyasaka, Sean N. Pike, "Pushing the limits of NuSTAR detectors," Proc. SPIE 10709, High Energy, Optical, and Infrared Detectors for Astronomy VIII, 107092V (9 August 2018); doi: $10.1117 / 12.2312844$

Event: SPIE Astronomical Telescopes + Instrumentation, 2018, Austin, Texas, United States 


\title{
Pushing the Limits of the NUSTAR Detectors
}

\author{
Brian W. Grefenstette ${ }^{\mathrm{a}}$, W. Rick Cook ${ }^{\mathrm{a}}$, Fiona A. Harrison ${ }^{\mathrm{a}}$, Takao Kitaguchi ${ }^{\mathrm{b}}$, Kristin K. \\ Madsen $^{\mathrm{a}}$, Hiromasa Miyasaka ${ }^{\mathrm{a}}$, and Sean N. Pike ${ }^{\mathrm{a}}$ \\ aSpace Radiation Lab, Caltech, 1200 E California Blvd, Pasadena, CA, USA \\ ${ }^{\mathrm{b}}$ RIKEN Nishina Center, 2-1 Hirosawa, Wako, Saitama 351-0198, Japan
}

\begin{abstract}
NuSTAR (the Nuclear Spectroscopic Telescope ARray) is a NASA Small Explorer (SMEX) mission launched in June of 2012. Since its launch, NuSTAR has been the preeminent instrument for spectroscopic analysis of the hard X-ray sky over the 3-80 keV bandpass. The low energy side of the bandpass is limited by the absorption along the photon path as well as by the ability of the pixels to trigger on incident photons. The on-board calibration source does not have a low-energy line that we can use to calibrate this part of the response, so instead we use the "nearest-neighbor" readout in the NuSTAR detector architecture to calibrate the individual pixel thresholds for all 8 flight detectors on both focal plane modules (FPMs). These threshold measurements feed back into the quantum efficiency of the detectors at low $(<5 \mathrm{keV})$ energies and, once well-calibrated, may allow the use of NuSTAR data below the current $3 \mathrm{keV}$ limit.
\end{abstract}

Keywords: Manuscript format, template, SPIE Proceedings, LaTeX

\section{INTRODUCTION}

The advent of imaging hard X-ray telescopes has brought a revolution in the sensitive study of the X-ray sky at energies beyond $10 \mathrm{keV}$. The two focusing optics flown on the Nuclear Spectroscopic Telescope ARray $($ NuSTAR $)$ NASA Astrophysics Small Explorer ${ }^{1}$ provide broad-band reflectivity across the hard X-ray bandpass. Each focus down on a focal plane of Cadium Zinc Telluride (CZT) read out by a custom, low-noise application specific integrated circuit (ASIC) developed at Caltech. ${ }^{2,3}$

At high energies, the bandpass of $N U S T A R$ is limited by the $\sim 78.4 \mathrm{keV}$ cutoff in the X-ray optics. ${ }^{4}$ At low energies the throughput drops from several main effects: (1) attenuation of the X-rays along the optical path due to absorbing materials; (2) attenuation in the detectors themselves; and (3) the ability of the pixels to trigger. For science analyses, all three of these effects area accounted for in the response files for the telescope (the "ancillary response file" or ARF accounts for the (1) and (2) while the "response matrix file" or RMF accounts for (3) as well as the off-diagonal response of the CZT detectors to incident high-energy photons).

In this paper we measure the trigger thresholds for the NuSTAR flight detectors on a pixel-by-pixel basis using in-flight calibration source data. We discuss our results, their impact on science observations, and the future plans for incorporating this calibration measurement into the NuSTAR response files.

\section{METHODS}

\subsection{Analysis}

NuSTAR has a unique readout architecture that allows us to directly measure the charge collected by a pixel even when the pixel has collected less charge than it requires to trigger. When an ASIC pixel collects charge, it is sent to the "fast" trigger chain which has a shaping a time of a few $\mu \mathrm{s}$. If this signal exceeds the threshold value then the full readout of the event is triggered.

The trigger threshold it set by a single control value for each CZT detector. This value is typically tuned to prevent an overabundance of "noise" triggers from a detector. Any variations in the gain of the "fast" chain

High Energy, Optical, and Infrared Detectors for Astronomy VIII, edited by Andrew D. Holland,

James Beletic, Proc. of SPIE Vol. 10709, 107092V · @ 2018 SPIE

CCC code: $0277-786 \mathrm{X} / 18 / \$ 18 \cdot$ doi: $10.1117 / 12.2312844$ 


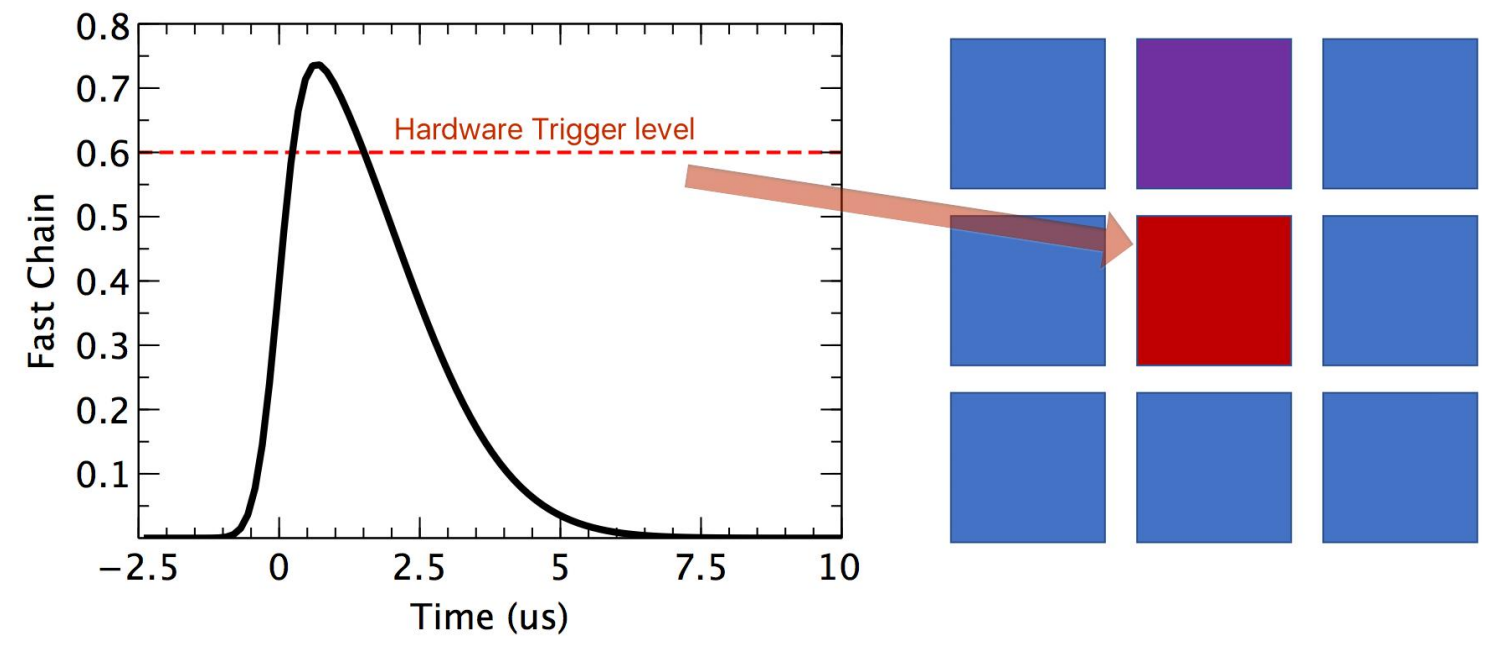

Figure 1. (Left) A schematic view of signal arriving at the "fast trigger" chain. When the signal exceeds the pixel hardware trigger threshold (dashed red line) the ASIC reads out a 3x3 grid of pulse heights (Right) centered on the triggered pixel. This allows us to study whether or not a neighboring pixel (here shown in purple) has a hardware trigger.

over the detector and/or differences in the detector-to-detector discriminator settings can result in differences in the trigger threshold level.

Once a pixel exceeds its threshold value, the front-end electronics reads out a 3x3 grid of pixels, centered on the pixel that collects the most charge (the red pixel in Figure 1). Hereafter we refer to this as the "central pixel". Typically (for about half of the events), most of the charge is collected by the central pixel. The information from the surrounding pixels is then used to improve the energy resolution and to reject background events. ${ }^{5}$ If a photon hits close to the edge of a pixel, then the charge cloud can drift onto a neighboring pixel. In this case the readout of the neighboring pixels is critical to reconstructing the total energy of the events and can be used to improve the spatial localization of events. ${ }^{6}$ As the photon arrives at different distances from the pixel edge we expect to see a relatively smooth continuum of "shared" charge with the neighboring pixel. Crucially, we get this information whether or not the neighboring pixels exceeds its on-board hardware trigger, though we do record diagnostic information that tells us the number of triggered pixels. We use the fact that the neighboring pixel effectively sees a smooth continuum of "shared" charge to measure the trigger threshold of the neighboring pixel.

First, we construct a "triggered spectrum" of the charge (here converted to an equivalent photon energy given in $\mathrm{keV}$ ) collected by a pixel that is a neighboring to the central pixel (the purple pixel in Figure 1). We chose events where there were exactly two hardware triggers (which we assume to be from both the purple and red pixels in Figure 1). We then compare this to the spectrum of all the events (Figure 3). By taking the ratio of these two spectra we obtain the hardware trigger probability as a function of energy. If the electronics were noise-free, then this ratio would be a step function with the step located at the threshold energy. In real detectors, such a measurement also has to contend with things such as electronic noise. This changes the functional form from a step function into an integrated Gaussian (i.e. the "error function") which can be parameterized by a mean energy (the threshold) and the width of the Gaussian (the measure of the electronic noise on the trigger line).

\subsection{Data Selection and Example of Data Quality}

NuSTAR deployed its on-board calibration source $\left({ }^{155} \mathrm{Eu}\right.$, which has prominent emission lines at 86 and $105 \mathrm{keV}$ as well as other emission lines below $40 \mathrm{keV}$ ) in January of 2015. For each pixel on the NuSTAR focal plane module (FPM) we extract all of the events where the central pixel of an event (i.e. the red pixel) is a neighbor of

Further author information: (Send correspondence to B.W.G)

E-mail: bwgref@srl.calech.edu, Telephone: 1 626-395-8037 


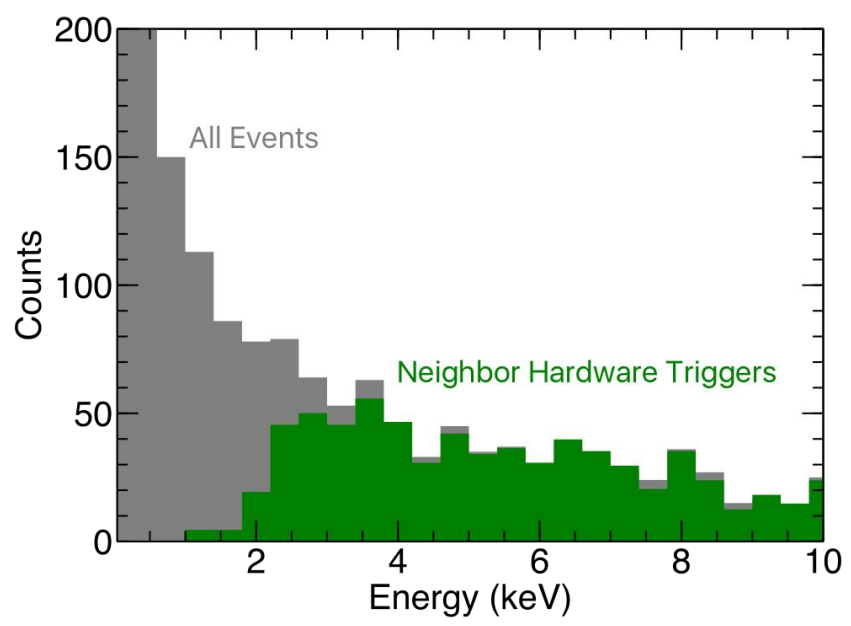

Figure 2. The energy spectrum of all events in the neighboring pixel in Figure 1 (gray) compared with those events where the neighboring pixel had a hardware trigger (green).

the pixel of interest (the purple pixel in Figure 1). In the $N u S T A R$ parlance, this refers to pixels with Grades 1-4, where the pixel of interest is offset by $(0,+1),(+1,0),(0,-1)$, and $(-1,0)$, respectively, in the (RAWX, RAWY) pixel coordinates from the central pixel. We use the PIS_GAIN column in the NuSTAR Level1A unfiltered event file as a proxy for the charge collected in the pixel, with the standard PI-to-keV scaling $\left(E_{k e V}=P I_{\text {channel }} \times 0.4+1.6\right)$. To accumulate sufficient statistics we also have to integrate over $3 \times 3$ regions on the detector, assuming that the trigger threshold gain will not vary substantially for nieghboring pixels. Because of data selection effects, the "hardware trigger" and "All Events" spectra shown in Figure 2 don't necessarily sum to unity in each energy bin. We therefore scale the ratio so that it represents unity over the 8-10 keV band (where we expect all pixels to trigger) and then fit the resulting ratio distribution with an integrated Gaussian function (Figure 3) using the curve_fit routines in SciPy. ${ }^{7}$

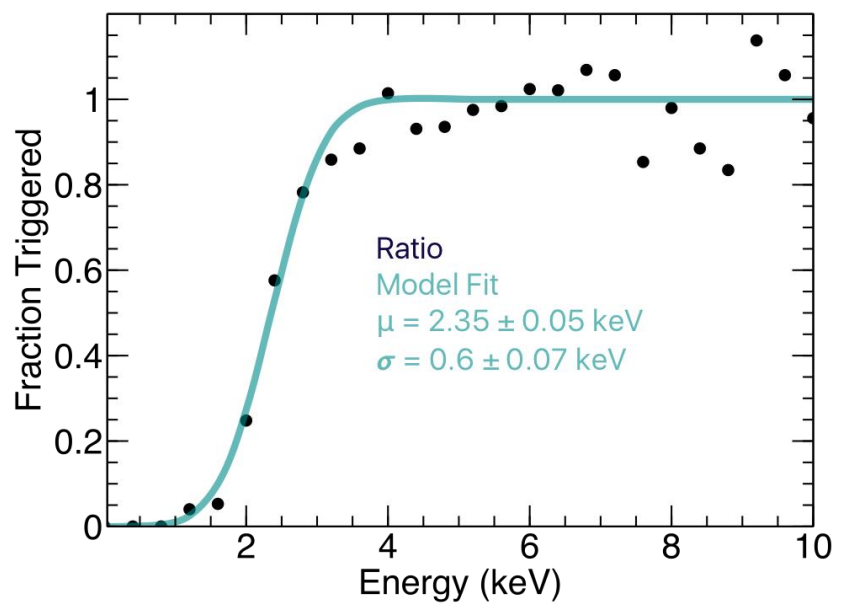

Figure 3. An example from one $3 \times 3$ region showing the ratio of hardware triggers to total events (black) and the fit of the integrated Gaussian to the data (dark cyan). The threshold energy $(\mu)$ and the Gaussian width $(\sigma)$ are both given along with 1- $\sigma$ error bars on each parameter. 


\section{RESULTS}

\subsection{NuSTAR FPM Results}

We can easily see that the there is both spatial variability on each detector as well as significant systematic differences between the four detectors on each FPM (Figure 4). The differences between pixels on a given detector are caused by the differences in the gain of the pixel threshold electronics, while the differences in the mean threshold value between different detectors actually reflect differences in how the detectors were configured on orbit.

During in-orbit commissioning, DET0 (top right in both FPM images) had its threshold set to a slightly higher energy to reduce the number of "noise" triggers on that detector. The other three detectors used the threshold values chosen during the ground calibration campaign chosen to balance the desire for a low-energy threshold against the desire for a low number of "noisy" pixels that had to be deactivated.
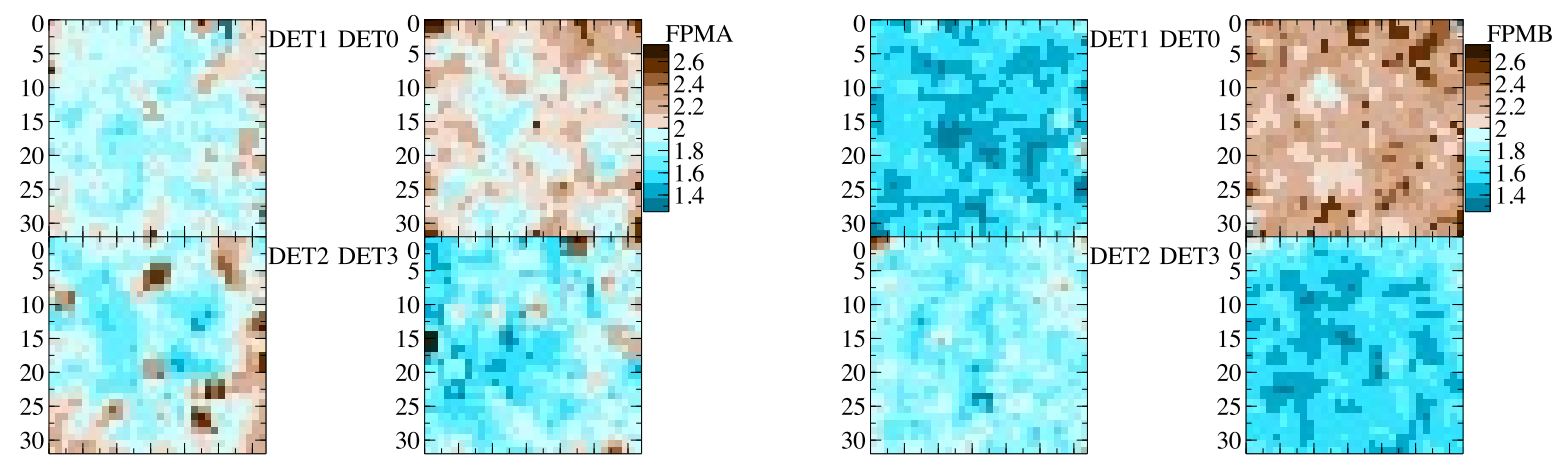

Figure 4. Color map representation of the threshold $(\mu)$ values for all of the pixels on both FPMA (left) and FPMB (right) on NuSTAR. The color map is common between all images and extends from $1.4 \mathrm{keV}$ (dark blue) to $2.6 \mathrm{keV}$ (dark brown).

For FPMA, the spread across each detector is larger than for FPMB, with the mean of the DET0 distribution only being marginally higher in energy than the distributions for the other three detectors. For FPMB, the distribution for each detector is much narrower and the clear shift of DET0 to higher energies compared with the threshold values for the other three detectors (especially DET1 and DET3) is easy to see by eye.

\subsection{Representation in the NuSTAR RMF}

For science analysis, the important factor here is the comparison of the energy threshold in the data with the expectation from the response files. All eight NuSTAR detectors have RMFs based on Monte Carlo simulations of the detectors that include the differences in the detector thicknesses and the charge transport properties in the detector, ${ }^{5}$ however the simulation assumes that all eight detectors have a threshold value of $2 \mathrm{keV}$. The left panel on Figure 6 shows the comparison of the measured-vs-expected values of the trigger threshold on the quantum efficiency of the detectors at low energies. In practice, analysis software assumes that the red curve is correct, which can introduced systematic errors. The left and right panels of Figure 6 show the level of this effect.

The nominal "calibrated" range for NuSTAR is $3-78.4 \mathrm{keV} .{ }^{4}$ Above $3 \mathrm{keV}$, the effect of the mismatch is relatively mild. For pixels with a threshold above $2 \mathrm{keV}$ (the cyan curve and pixels on DET0) this can result in an artificially drop below $4 \mathrm{keV}$ on the order of $5 \%$ at $3 \mathrm{keV}$. For pixels with a threshold substantially lower than $2 \mathrm{keV}$ (the purple curve in Figure 6 which is roughly the nominal value for DET1 and DET3 on FPMB), then this can result in an artificially overshoot the data below $4 \mathrm{keV}$, again with errors on the order of $5 \%$.

\subsection{Science Impact}

In practice, there are numerous detector-specific corrections to the NuSTAR response files that affect the throughput at low energies. Several of these are measured on a detector-by-detector basis (such as the detector absorption coefficients $^{4}$ ), which are likely to be compensating for (and degenerate with) the mismatch between the actual 


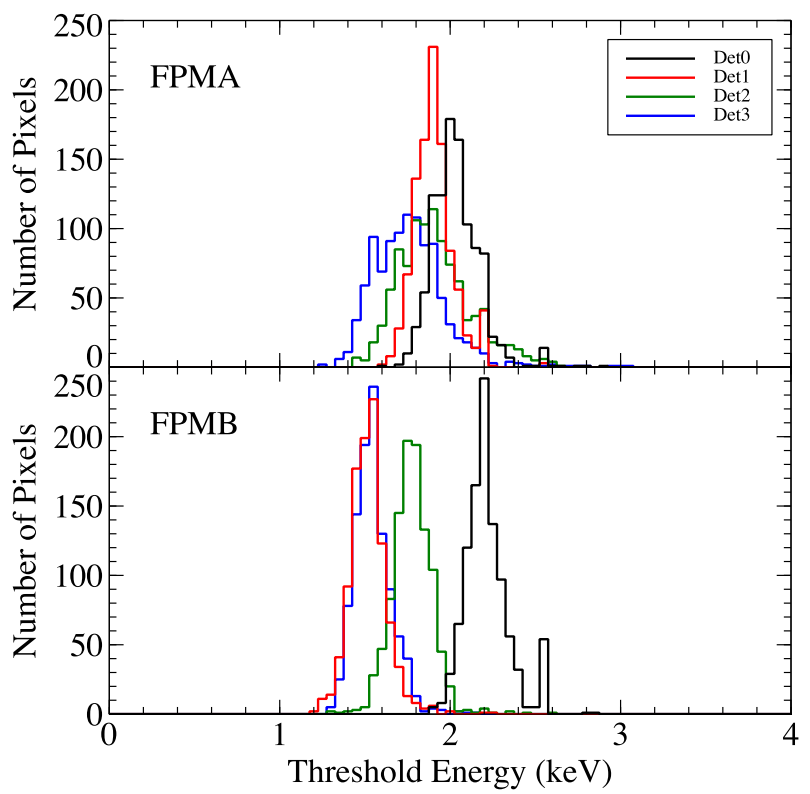

Figure 5. Histograms of the color map distributions shown in Figure 4 for the four NuSTAR detectors on FPMA (top) and FPMB (bottom). The systematic shift of DET0 to higher energies can be seen on both FPMs (though more clearly on FPMB).
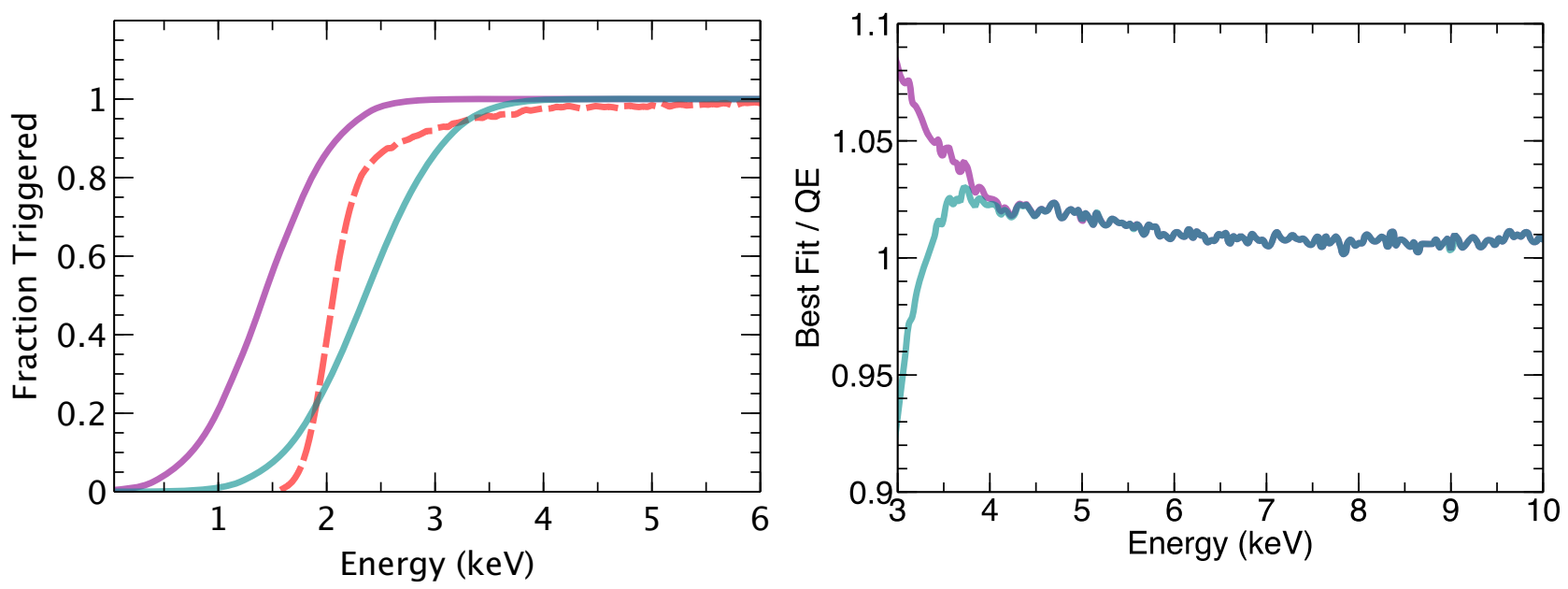

Figure 6. (Left) Comparison of the effect changing the trigger threshold value. The nominal threshold is shown as well as ( $2 \mathrm{keV}$, red) examples where the threshold exceeds this value $(2.35 \mathrm{keV}$, cyan) and is below this value (1.35 keV, purple). The impact of this over the nominal $N u S T A R$ energy range $(\mathrm{E}>3 \mathrm{keV})$ is shown as a ratio of of the purple/cyan curves in the left panel to the red curve in the left panel.

threshold and what is reported in the RMF. While we would prefer a first-principles approach to the production of the NuSTAR RMFs, we only anticipate an impact on the science for observations with significant signal-to-noise at low energies.

We do also note that DET1 and DET3 on FPMB have a threshold value substantially below the nominal 2 $\mathrm{keV}$ level. The NuSTAR RMF files were constructed using an energy grid with 4096 channels, each with a 40 $\mathrm{eV}$ width starting at $1.6 \mathrm{keV}$. For pixels with a threshold below this level we expect there to be a significant number of photons below $1.6 \mathrm{keV}$ that may be getting artificially vetoed by the NuSTAR pipeline (essentially 
for apparently having a negative channel number). If the $N u S T A R$ low-energy response $(\mathrm{E}<3 \mathrm{keV})$ can be wellcalibrated, then this would imply a further overlap with soft X-ray telescopes, extending the opportunities for joint science observations.

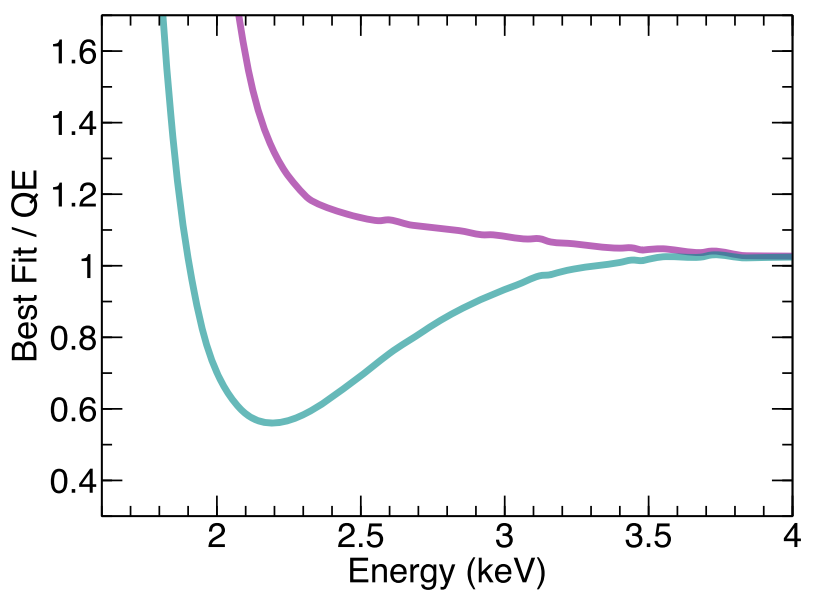

Figure 7. The same as for the right panel of Figure 6, but extrapolated to energies below the nominal NuSTAR calibrated range showing the large deviations resulting over the $2-3 \mathrm{keV}$ bandpass that may be calibrated out by using a more realistic energy threshold in the RMF simulation.

\subsection{Future Work}

In the future, we anticipate experimenting with re-producing the $N u S T A R$ RMF files and extending the $N u S T A R$ calibrated energy range. We do not anticipate changing the channel energy scale (40 eV per channel), but instead simply shifting the current energy range (which is defined to be from $1.6 \mathrm{keV}$ to $165.44 \mathrm{keV}$ for Channel 0 to 4095) down by $1 \mathrm{keV}$ (covering instead $0.6 \mathrm{keV}$ to $164.44 \mathrm{keV}$ ). The upper half of the NuSTAR energy band is essentially used for gain and particle background studies, so we do not expect that trimming one keV off of the upper edge to impact any analysis.

At low energies, we hope to fold in new NuSTAR RMF files for each NuSTAR detector using the mean $\mu$ and $\sigma$ parameters measured in this analysis, potentially allowing us to extend the usable science energy bandpass for observations where the source falls on DET1 and DEt3.

This work only used data from the 2015 calibration source campaign. We need to compare the 2012 calibration source measurements with those from 2015 to see if there are any time-dependent changes in the low-energy threshold. We also need to see if we can reasonably track the threshold as a function of time using data from other sources (i.e. background data sets and/or calibration observations of astrophysical sources) so that we do not require additional deployments of the calibration source to perform additional threshold measurements.

\section{CONCLUSIONS}

We report on new in-flight measurements on the low-energy threshold of the NuSTAR detectors. Leveraging the $N u S T A R$ readout electronics we are able to measure the energy threshold of small $3 \times 3$ clusters of pixels. We find that there is substantial disagreement between the threshold values used to produce the NuSTAR RMFs and those that we measure during the in-flight data. DET0 on both FPMs has a systematically higher low-energy threshold compared to the other three detectors, which we attribute to configuration of the detectors during early science operations. We do not, however, expect there to be a significant impact of this mis-match except for sources with extremely high signal-to-noise (where these systematic $\approx 5 \%$ effects can be seen over the Poisson noise or for observations that wish to extend their analysis of NuSTAR data below the nominal $3 \mathrm{keV}$ calibrated range. 


\section{ACKNOWLEDGMENTS}

BG acknowledges valuable conversations with W. Rick Cook and Jill Burhnam during the development of this paper. This work was supported under NASA Contract No. NNG08FD60C, and made use of data from the $N u S$ $T A R$ mission, a project led by the California Institute of Technology, managed by the Jet Propulsion Laboratory, and funded by the National Aeronautics and Space Administration. This work makes use of open source code produced by the community, including Astropy, a community-developed core Python package for Astronomy (Astropy Collaboration, 2018) and the SciPy ecosystem. All graphics were produced using Veusz, Copyright (C) 2003-2018 Jeremy Sanders and contributors.

\section{REFERENCES}

[1] Harrison, F. A., Craig, W. W., Christensen, F. E., Hailey, C. J., Zhang, W. W., Boggs, S. E., Stern, D., Cook, W. R., Forster, K., Giommi, P., Grefenstette, B. W., Kim, Y., Kitaguchi, T., Koglin, J. E., Madsen, K. K., Mao, P. H., Miyasaka, H., Mori, K., Perri, M., Pivovaroff, M. J., Puccetti, S., Rana, V. R., Westergaard, N. J., Willis, J., Zoglauer, A., An, H., Bachetti, M., Barrire, N. M., Bellm, E. C., Bhalerao, V., Brejnholt, N. F., Fuerst, F., Liebe, C. C., Markwardt, C. B., Nynka, M., Vogel, J. K., Walton, D. J., Wik, D. R., Alexander, D. M., Cominsky, L. R., Hornschemeier, A. E., Hornstrup, A., Kaspi, V. M., Madejski, G. M., Matt, G., Molendi, S., Smith, D. M., Tomsick, J. A., Ajello, M., Ballantyne, D. R., Balokovi, M., Barret, D., Bauer, F. E., Blandford, R. D., Brandt, W. N., Brenneman, L. W., Chiang, J., Chakrabarty, D., Chenevez, J., Comastri, A., Dufour, F., Elvis, M., Fabian, A. C., Farrah, D., Fryer, C. L., Gotthelf, E. V., Grindlay, J. E., Helfand, D. J., Krivonos, R., Meier, D. L., Miller, J. M., Natalucci, L., Ogle, P., Ofek, E. O., Ptak, A., Reynolds, S. P., Rigby, J. R., Tagliaferri, G., Thorsett, S. E., Treister, E., and Urry, C. M., "THE NUCLEAR SPECTROSCOPIC TELESCOPE ARRAY ( NuSTAR ) HIGH-ENERGY X-RAY MISSION," The Astrophysical Journal 770, 103 (May 2013).

[2] Cook, W. R., Boggs, S. E., Bolotnikov, A. E., Burnham, J. A., Fitzsimmons, M. J., Harrison, F. A., Kecman, B., Matthews, B., and Schindler, S. M., "High resolution CdZnTe pixel detectors with VLSI readout," IEEE Transactions on Nuclear Science 47, 1454-1457 (Aug. 2000).

[3] Rana, V. R., Cook, W. R., Harrison, F. A., Mao, P. H., and Miyasaka, H., "Development of focal plane detectors for the Nuclear Spectroscopic Telescope Array (NuSTAR) mission," UV, X-Ray, and Gamma-Ray Space Instrumentation for Astronomy XVI 7435, 743503 (Aug. 2009).

[4] Madsen, K. K., Harrison, F. A., Markwardt, C. B., An, H., Grefenstette, B. W., Bachetti, M., Miyasaka, H., Kitaguchi, T., Bhalerao, V., Boggs, S., Christensen, F. E., Craig, W. W., Forster, K., Fuerst, F., Hailey, C. J., Perri, M., Puccetti, S., Rana, V., Stern, D., Walton, D. J., Jrgen Westergaard, N., and Zhang, W. W., "Calibration of the NuSTAR High-energy Focusing X-ray Telescope.," The Astrophysical Journal Supplement Series 220, 8 (Sept. 2015).

[5] Kitaguchi, T., Grefenstette, B. W., Harrison, F. A., Miyasaka, H., Bhalerao, V. B., Cook, W. R., Mao, P. H., Rana, V. R., Boggs, S. E., and Zoglauer, A. C., "Spectral calibration and modeling of the NuSTAR CdZnTe pixel detectors," Society of Photo-Optical Instrumentation Engineers (SPIE) Conference Series 8145, 814507 (Sept. 2011).

[6] Grefenstette, B. W., Bhalerao, V., Cook, W. R., Harrison, F. A., Kitaguchi, T., Madsen, K. K., Mao, P. H., Miyasaka, H., and Rana, V., "Ground calibration of the spatial response and quantum efficiency of the CdZnTe hard x-ray detectors for NuSTAR," Society of Photo-Optical Instrumentation Engineers (SPIE) Conference Series 10392, 1039207 (Aug. 2017).

[7] Jones, E., Oliphant, T., Peterson, P., and others, [SciPy: Open source scientific tools for Python] (2001). 